TRANSACTIONS OF THE

AMERICAN MATHEMATICAL SOCIETY

Volume 361, Number 10, October 2009, Pages 5555-5574

S 0002-9947(09)04739-4

Article electronically published on May 20, 2009

\title{
SHARP CONSTANTS RELATED TO THE TRIANGLE INEQUALITY IN LORENTZ SPACES
}

\author{
SORINA BARZA, VIKTOR KOLYADA, AND JAVIER SORIA
}

Abstract. We study the Lorentz spaces $L^{p, s}(R, \mu)$ in the range $1<p<s \leq$ $\infty$, for which the standard functional

$$
\|f\|_{p, s}=\left(\int_{0}^{\infty}\left(t^{1 / p} f^{*}(t)\right)^{s} \frac{d t}{t}\right)^{1 / s}
$$

is only a quasi-norm. We find the optimal constant in the triangle inequality for this quasi-norm, which leads us to consider the following decomposition norm:

$$
\|f\|_{(p, s)}=\inf \left\{\sum_{k}\left\|f_{k}\right\|_{p, s}\right\},
$$

where the infimum is taken over all finite representations $f=\sum_{k} f_{k}$. We also prove that the decomposition norm and the dual norm

$$
\|f\|_{p, s}^{\prime}=\sup \left\{\int_{R} f g d \mu:\|g\|_{p^{\prime}, s^{\prime}}=1\right\}
$$

agree for all values of $p, s>1$.

\section{INTRODUCTION}

The study of the normability of the Lorentz spaces $L^{p, s}(R, \mu)$ goes back to the work of G.G. Lorentz [10, 11] (see also [13, 3, 2] for a more recent account of the normability results for weighted Lorentz spaces). The condition defining these spaces is given in terms of the distribution function and, equivalently, the nonincreasing rearrangement of $f$ (see [1] for standard notation and basic definitions):

$$
\|f\|_{p, s}=\left(\int_{0}^{\infty}\left(t^{1 / p} f^{*}(t)\right)^{s} \frac{d t}{t}\right)^{1 / s},
$$

with the usual modification if $s=\infty$. Lorentz proved that \|\|$_{p, s}$ is a norm if and only if $1 \leq s \leq p<\infty$, and that the space $L^{p, s}(R, \mu)$ is always normable (i.e., there exists a norm equivalent to \|\|$_{p, s}$ ) for the range $1<p<s \leq \infty$ (for the remaining cases it is known that $L^{p, s}(R, \mu)$ cannot be endowed with an equivalent norm). From now on we will only consider the range of indices $1<p<\infty, 1 \leq s \leq \infty$.

Received by the editors June 25, 2007 and, in revised form, January 8, 2008.

2000 Mathematics Subject Classification. Primary 46E30, 46B25.

Key words and phrases. Equivalent norms, level function, Lorentz spaces, sharp constants.

An essential part of this work was performed while the first and second authors stayed at the University of Barcelona as invited researchers. We express our gratitude to the Department of Mathematics of the University of Barcelona for the hospitality and excellent conditions.

The third author was partially supported by grants MTM2007-60500 and 2005SGR00556.

(C)2009 American Mathematical Society Reverts to public domain 28 years from publication 
Note that the spaces $L^{p, s}$, with $p<s$, play an important role not only as dual spaces for the Banach spaces $L^{p^{\prime}, s^{\prime}}$ (see [1, 7]). For example, they also arise naturally in limiting embeddings of Lipschitz spaces $([8])$.

The study of the normability for $p<s$ was carried out by means of the maximal norm:

where

$$
\|f\|_{p, s}^{*}=\left(\int_{0}^{\infty}\left(t^{1 / p} f^{* *}(t)\right)^{s} \frac{d t}{t}\right)^{1 / s}
$$

$$
f^{* *}(t)=\frac{1}{t} \int_{0}^{t} f^{*}(x) d x .
$$

It is easy to see that \|\|$_{p, s}^{*}$ is always a norm. Moreover, one can prove that \|\|$_{p, s}^{*}$ is equivalent to \|\|$_{p, s}$, with the following optimal estimates:

$$
\left(p^{\prime}\right)^{1 / s}\|f\|_{p, s} \leq\|f\|_{p, s}^{*} \leq p^{\prime}\|f\|_{p, s}
$$

(see [14, 9]; as usual, $p^{\prime}$ denotes the conjugate exponent, $1 / p+1 / p^{\prime}=1$ ).

As a consequence of the fact that \|\|$_{p, s}$ is equivalent to a norm, it is easy to see that it is a quasi-norm satisfying the triangle inequality, uniformly on the number of terms: there exists a constant $c_{p, s}>0$ such that for every finite collection $\left\{f_{k}\right\}_{k=1, \cdots, N} \subset L^{p, s}(R, \mu)$,

$$
\left\|\sum_{k=1}^{N} f_{k}\right\|_{p, s} \leq c_{p, s} \sum_{k=1}^{N}\left\|f_{k}\right\|_{p, s} .
$$

The converse result can readily be proved; namely, (1.2) is equivalent to the fact that \|\|$_{p, s}$ is normable and, even more, that an alternative equivalent norm is given by means of the following decomposition norm:

$$
\|f\|_{(p, s)}=\inf \left\{\sum_{k}\left\|f_{k}\right\|_{p, s}\right\},
$$

where the infimum is taken over all finite representations $f=\sum_{k} f_{k}$.

It is easy to prove that \|\|$_{(p, s)}$ is a norm, equivalent to \|\|$_{p, s}$, that agrees with \|\|$_{p, s}$ if $1 \leq s \leq p$. Moreover, the best constant in the inequality $\|f\|_{p, s} \leq$ $c_{p, s}\|f\|_{(p, s)}$ is the same as the optimal one in (1.2). One of the main problems studied in this paper is to find the best constant in the triangle inequality (1.2) and its continuous version, the Minkowski integral inequality (the control of these constants is sometimes very relevant for estimating different types of integral operators, where the use of the maximal norm and the inequalities (1.1) do not usually give optimal results).

For the Lorentz norms we have the following version of Hölder's inequality: if $f \in L^{p, s}(R, \mu)$ and $g \in L^{p^{\prime}, s^{\prime}}(R, \mu)(1<p<\infty, 1 \leq s \leq \infty)$, then

$$
\left|\int_{R} f g d \mu\right| \leq\|f\|_{p, s}\|g\|_{p^{\prime}, s^{\prime}}
$$

(see [1, p. 220]).

In the theory of Banach function spaces $\left(L^{p, s}(R, \mu)\right.$ being the canonical example in this context) and based on (1.4), it is also very natural to consider another norm defined in terms of the Köthe duality, which is denoted as the dual norm:

$$
\|f\|_{p, s}^{\prime}=\sup \left\{\int_{R} f g d \mu:\|g\|_{p^{\prime}, s^{\prime}}=1\right\} \text {. }
$$


As in the case of the decomposition norm, \|\|$_{p, s}^{\prime}$ is a norm, equivalent to \|\|$_{p, s}$ with $\|f\|_{p, s}^{\prime}=\|f\|_{p, s}$, if $1 \leq s \leq p$ (see (4.5) ). Therefore, $\|f\|_{p, s}^{\prime}=\|f\|_{(p, s)}(1 \leq s \leq p)$.

The main result that we will prove in this paper shows that the decomposition and dual norms agree over the whole range of indices (Theorem 5.2), in spite of their quite different definitions. We also find the best constants in the inequalities relating either of these norms and \|\|$_{p, s}$ (see (4.4), Theorem 4.4, and Remark 4.3). In particular, these results give an alternative proof of the normability of $L^{p, s}(R, \mu)$ with optimal estimates. We would like to remark that, while (1.1) follows easily from standard estimates, finding the best constants in our context requires new ideas and much more complicated constructions.

In Section 2 we prove several technical lemmas used in subsequent sections. Section 3 introduces one of the key tools used in the paper: the level function (see Theorems 3.1 and 3.2). Sections 4 and 5 are the core of the paper, dealing with both the dual and decomposition norms, and proving the main results already mentioned above. Finally, in Section 6 we obtain the best constant in both the triangle and Minkowski's integral inequalities for the Lorentz spaces.

Throughout this paper $(R, \mu)$ denotes a $\sigma$-finite nonatomic measure space.

\section{Auxiliary propositions}

In this section we consider some auxiliary results that will be used in what follows. We begin with some general inequalities.

Lemma 2.1. Let $f$ and $g$ be non-increasing nonnegative functions on $[0,1]$. Then

$$
\int_{0}^{1} f(x) d x \int_{0}^{1} g(x) d x \leq \int_{0}^{1} f(x) g(x) d x .
$$

This is the classical Chebyshev inequality (see, e.g., [6]).

Corollary 2.2. Let $g$ be a non-increasing nonnegative function on $[0,1]$ and let $0<\alpha<1$. Then

$$
\int_{0}^{1} g(x) d x \leq(1-\alpha) \int_{0}^{1} g(x) x^{-\alpha} d x .
$$

Lemma 2.3. Let $p, s \in(1, \infty)$. Then for any $t \in[0,1]$,

$$
\left(1-t^{s / p}\right)^{1 / s}\left(1-t^{s^{\prime} / p^{\prime}}\right)^{1 / s^{\prime}} \leq 1-t .
$$

Proof. We will prove that for all $x, y \in(0,1)$,

$$
\left(1-x^{s}\right)^{1 / s}\left(1-y^{s^{\prime}}\right)^{1 / s^{\prime}} \leq 1-x y .
$$

Then (2.2) will follow from (2.3) if we take $x=t^{1 / p}, y=t^{1 / p^{\prime}}$. To prove (2.3), fix $y$ and let

$$
\varphi(x)=1-x y-\left(1-x^{s}\right)^{1 / s}\left(1-y^{s^{\prime}}\right)^{1 / s^{\prime}} .
$$

We have

$$
\varphi^{\prime}(x)=-y-\frac{x^{s-1}}{\left(1-x^{s}\right)^{1 / s^{\prime}}}\left(1-y^{s^{\prime}}\right)^{1 / s^{\prime}} .
$$

Set $\varphi^{\prime}(x)=0$. Then

$$
\frac{\left(1-x^{s}\right)^{1 / s^{\prime}}}{x^{s-1}}=\frac{\left(1-y^{s^{\prime}}\right)^{1 / s^{\prime}}}{y}
$$


and

$$
\left(\frac{1}{x^{s}}-1\right)^{1 / s^{\prime}}=\left(\frac{1}{y^{s^{\prime}}}-1\right)^{1 / s^{\prime}} .
$$

This implies that $x^{s}=y^{s^{\prime}}$; hence the function $\varphi$ has an absolute minimum for $x=y^{1 /(s-1)}$ and this minimum is 0 , which proves (2.3).

The following lemma gives the sharp constant in the relation between Lorentz norms with different second indices (see [14, p. 192]).

Lemma 2.4. Let $1 \leq p<\infty$ and $1 \leq r<s \leq \infty$. Then, for any function $f \in L^{p, r}(R, \mu)$,

$$
\left(\frac{s}{p}\right)^{1 / s}\|f\|_{p, s} \leq\left(\frac{r}{p}\right)^{1 / r}\|f\|_{p, r} .
$$

We consider now some auxiliary statements related to the dual norm and decomposition norm.

Lemma 2.5. Let $f \in L^{p, s}(R, \mu)(1<p<\infty, 1 \leq s \leq \infty)$. Then

$$
\|f\|_{p, s}^{\prime}=\left\|f^{*}\right\|_{p, s}^{\prime}
$$

The proof can be found in [1, p. 45-49].

Lemma 2.6. Let $f \in L^{p, s}(R, \mu)(1<p<\infty, 1 \leq s \leq \infty)$. Then

$$
\|f\|_{p, s}^{\prime} \leq\|f\|_{(p, s)} .
$$

Proof. Let $g \in L^{p^{\prime}, s^{\prime}}(R, \mu)$ and let

$$
f=\sum_{k} f_{k}
$$

Then, by Hölder's inequality (1.4),

$$
\int_{R}|f g| d \mu \leq \sum_{k} \int_{R}\left|f_{k} g\right| d \mu \leq\|g\|_{p^{\prime}, s^{\prime}} \sum_{k}\left\|f_{k}\right\|_{p, s} .
$$

Taking the infimum over all representations (2.7), we obtain (2.6).

We shall use the following properties of the decomposition norm.

Lemma 2.7. Let $f \in L^{p, s}(R, \mu)(1<p<\infty, 1 \leq s \leq \infty)$. Then:

(1) the equality

$$
\|f\|_{(p, s)}=\inf \left\{\sum_{k}\left\|f_{k}\right\|_{p, s}\right\}
$$

holds, where the infimum is taken over all finite sequences $\left\{f_{k}\right\}$ such that $f_{k} \geq 0$ and

$$
|f(x)|=\sum_{k} f_{k}(x)
$$

(2) if $0 \leq g \leq f$, then $\|g\|_{(p, s)} \leq\|f\|_{(p, s)}$;

(3) if $0 \leq g_{n} \leq f$ and $g_{n}(x) \uparrow f(x) \mu$-almost everywhere on $R$, then $\left\|g_{n}\right\|_{(p, s)} \rightarrow$ $\|f\|_{(p, s)}$. 
Proof. Denote by $\sigma$ the right hand side of (2.8). We have $f=\sum_{k} g_{k}$, where $g_{k}=f_{k} \operatorname{sign} f$, and therefore

$$
\|f\|_{(p, s)} \leq \sum_{k}\left\|g_{k}\right\|_{p, s}=\sum_{k}\left\|f_{k}\right\|_{p, s}
$$

Thus, $\|f\|_{(p, s)} \leq \sigma$. On the other hand, for any $\varepsilon>0$ there exists a representation $f=\sum_{k} g_{k}$ such that

$$
\|f\|_{(p, s)}>\sum_{k}\left\|g_{k}\right\|_{p, s}-\varepsilon .
$$

We have $|f| \leq \sum_{k}\left|g_{k}\right| \equiv G$. Set $f_{k}=\left|f g_{k}\right| / G$. Then $\left\|f_{k}\right\|_{p, s} \leq\left\|g_{k}\right\|_{p, s}$ and $|f|=\sum_{k} f_{k}$. Thus $\|f\|_{(p, s)} \geq \sigma-\varepsilon$, which proves (2.8). Further, statement (2) follows immediately from statement (1). To prove (3), observe that $\left\|f-g_{n}\right\|_{p, s} \rightarrow 0$ (see [1, p. 41]). Since

$$
\left\|g_{n}\right\|_{(p, s)} \leq\|f\|_{(p, s)} \leq\left\|g_{n}\right\|_{(p, s)}+\left\|f-g_{n}\right\|_{p, s},
$$

we obtain (3).

Lemma 2.8. For each $f \in L^{p, s}(R, \mu)$,

$$
\|f\|_{(p, s)} \leq\left\|f^{*}\right\|_{(p, s)} .
$$

Proof. It is known that there exists a measure preserving transformation $\sigma: R \rightarrow$ $(0, \mu(R))$ such that

$$
f(x)=f^{*}(\sigma(x)), \quad \mu \text {-a.e. on } \quad R,
$$

(see [1, p. 82, 83]). Let $f^{*}(t)=\sum_{k=1}^{N} g_{k}(t), g_{k} \geq 0$. Then $f(x)=\sum_{k=1}^{N} g_{k}(\sigma(x))$. Since $g_{k} \circ \sigma$ and $g_{k}$ are equimeasurable, we have that

$$
\|f\|_{(p, s)} \leq \sum_{k=1}^{N}\left\|g_{k} \circ \sigma\right\|_{p, s}=\sum_{k=1}^{N}\left\|g_{k}\right\|_{p, s} .
$$

This implies (2.9).

It will be proved below that for any $f$ we have the equality in (2.9).

Lemma 2.9. Let $1<p<\infty$. Assume that $f \in L^{p, s_{0}}(R, \mu)$ for some $p \leq s_{0}<\infty$. Then

$$
\|f\|_{p, \infty}=\lim _{s \rightarrow \infty}\|f\|_{p, s}
$$

and

$$
\|f\|_{(p, \infty)}=\lim _{s \rightarrow \infty}\|f\|_{(p, s)} .
$$

Proof. To prove (2.11), we can assume that $\mu(\operatorname{supp} f)<\infty$. Then (2.11) follows from a similar property for the $L^{s}$-norm (see [4, p. 226]).

We shall prove (2.12). By Lemma 2.7, we can assume that $f \geq 0$ and consider only representations

$$
f=\sum_{k=1}^{N} f_{k}, \quad \text { where } \quad f_{k} \geq 0 .
$$


For an arbitrary representation (2.13) we have that, for any $s>s_{0}$,

$$
\|f\|_{(p, s)} \leq \sum_{k=1}^{N}\left\|f_{k}\right\|_{p, s}
$$

By (2.11), we obtain that

$$
\varlimsup_{s \rightarrow \infty}\|f\|_{(p, s)} \leq \sum_{k=1}^{N}\left\|f_{k}\right\|_{p, \infty}
$$

which implies that

$$
\varlimsup_{s \rightarrow \infty}\|f\|_{(p, s)} \leq\|f\|_{(p, \infty)} .
$$

To prove the reverse inequality, take an arbitrary $\varepsilon>0$. For a fixed $s>s_{0}$, find a decomposition (2.13) such that

$$
\|f\|_{(p, s)}>\sum_{k=1}^{N}\left\|f_{k}\right\|_{p, s}-\varepsilon
$$

Applying inequality (2.4), we obtain

$$
\begin{aligned}
\|f\|_{(p, s)} & >\left(\frac{p}{s}\right)^{1 / s} \sum_{k=1}^{N}\left\|f_{k}\right\|_{p, \infty}-\varepsilon \\
& >\sum_{k=1}^{N}\left\|f_{k}\right\|_{p, \infty}-\varepsilon \geq\|f\|_{(p, \infty)}-\varepsilon .
\end{aligned}
$$

Thus, $\|f\|_{(p, s)}>\|f\|_{(p, \infty)}-\varepsilon$ for any $s>s_{0}$ and any $\varepsilon>0$. It follows that

$$
\varliminf_{s \rightarrow \infty}\|f\|_{(p, s)} \geq\|f\|_{(p, \infty)},
$$

which, together with (2.14), proves (2.12).

Lemma 2.10. Let $h(x)=\chi_{[0,1]}(x)$ and $1<p<\infty, 1 \leq s \leq \infty$. Then

$$
\|h\|_{p, s}=\left(\frac{p}{s}\right)^{1 / s} .
$$

If $p<s$, then

$$
\|h\|_{p, s}^{\prime}=\|h\|_{(p, s)}=\left(\frac{s^{\prime}}{p^{\prime}}\right)^{1 / s^{\prime}} .
$$

Proof. The equality (2.15) is immediate. We shall prove (2.16). Write $\alpha=1-s^{\prime} / p^{\prime}$ and set

$$
\varphi(t)=(1-\alpha) t^{-\alpha}, \quad t \in(0,1] .
$$

We have

$$
\left\|\varphi \chi_{[0,1]}\right\|_{p, s}=\left(\frac{s^{\prime}}{p^{\prime}}\right)^{1 / s^{\prime}}
$$


To evaluate the dual norm of $h$, we assume that $g \in L^{p^{\prime}, s^{\prime}}\left(\mathbb{R}_{+}\right), g \geq 0$ and $\|g\|_{p^{\prime}, s^{\prime}}=$ 1. Applying (2.1), Hölder's inequality (1.4), and (2.18), we obtain

$$
\begin{aligned}
\int_{\mathbb{R}_{+}} h(x) g(x) d x & \leq \int_{0}^{1} g^{*}(x) d x \\
& \leq(1-\alpha) \int_{0}^{1} g^{*}(x) x^{-\alpha} d x \leq\|g\|_{p^{\prime}, s^{\prime}}\|\varphi\|_{p, s}=\left(\frac{s^{\prime}}{p^{\prime}}\right)^{1 / s^{\prime}} .
\end{aligned}
$$

On the other hand, if

$$
g(x)=\left(\frac{s^{\prime}}{p^{\prime}}\right)^{1 / s^{\prime}} \chi_{[0,1]}(x)
$$

then $\|g\|_{p^{\prime}, s^{\prime}}=1$ and

$$
\int_{\mathbb{R}_{+}} h(x) g(x) d x=\left(\frac{s^{\prime}}{p^{\prime}}\right)^{1 / s^{\prime}} .
$$

Thus,

$$
\|h\|_{p, s}^{\prime}=\left(\frac{s^{\prime}}{p^{\prime}}\right)^{1 / s^{\prime}}
$$

We now prove the second equality in (2.16) (in Section 5 we shall prove that the dual and the decomposition norms always agree, but the proof of this fact for a characteristic function is much simpler). Let $1<p<s<\infty$. Assume that the function $\varphi$ in (2.17) is extended to the whole line $\mathbb{R}$ periodically with period 1 . Set

$$
g_{N}(x)=N \int_{x}^{x+1 / N} \varphi(t) d t
$$

Then $g_{N}(x) \rightarrow \varphi(x)$ as $N \rightarrow \infty$ for all $x \in(0,1)$. Moreover,

$$
\left(g_{N} \chi_{[0,1]}\right)^{*}(t) \leq\left(g_{N} \chi_{[0,1]}\right)^{* *}(t) \leq \varphi^{* *}(t)=t^{-\alpha}, \quad t \in(0,1] .
$$

Applying Lebesgue's dominated convergence theorem and (2.18), we obtain

$$
\left\|g_{N} \chi_{[0,1]}\right\|_{p, s} \rightarrow\left\|\varphi \chi_{[0,1]}\right\|_{p, s}=\left(\frac{s^{\prime}}{p^{\prime}}\right)^{1 / s^{\prime}}
$$

Let $\varepsilon>0$. Fix a number $N$ such that

$$
\left\|g_{N} \chi_{[0,1]}\right\|_{p, s}<\left(\frac{s^{\prime}}{p^{\prime}}\right)^{1 / s^{\prime}}+\varepsilon
$$

Set

$$
f_{k}(x)=\int_{(k-1) / N}^{k / N} \varphi(x+t) d t=\frac{1}{N} g_{N}\left(x+\frac{k-1}{N}\right), \quad k=1, \ldots, N .
$$

Then

$$
\sum_{k=1}^{N} f_{k}(x)=\int_{0}^{1} \varphi(x+t) d t=1,
$$

for all $x$. Since $f_{k}$ are 1-periodic and $\left.f_{k}(x)=f_{1}(x+(k-1) / N)\right)$, the restrictions of $f_{k}$ to $[0,1]$ are pairwise equimeasurable. Now set

$$
h_{k}(x)=f_{k}(x) \chi_{[0,1]}(x), \quad k=1, \ldots, N
$$


Then, by (2.21), $h=\sum_{k=1}^{N} h_{k}$ and, by (2.20),

$$
\sum_{k=1}^{N}\left\|h_{k}\right\|_{p, s}<\left(\frac{s^{\prime}}{p^{\prime}}\right)^{1 / s^{\prime}}+\varepsilon
$$

This implies that

$$
\|h\|_{(p, s)} \leq\left(\frac{s^{\prime}}{p^{\prime}}\right)^{1 / s^{\prime}}, \quad \text { for } \quad p<s<\infty .
$$

By Lemma 2.9. (2.22) holds for all $p<s \leq \infty$. The opposite inequality follows from (2.19) and Lemma 2.6.

We shall use the following lemma of Hardy [1, p. 56].

Lemma 2.11. Let $f_{1}$ and $f_{2}$ be nonnegative measurable functions on $\mathbb{R}_{+}$such that

$$
\int_{0}^{t} f_{1}(u) d u \leq \int_{0}^{t} f_{2}(u) d u
$$

for all $t>0$. Then, for every nonnegative and non-increasing function $g$ on $\mathbb{R}_{+}$, we have that

$$
\int_{0}^{\infty} f_{1}(u) g(u) d u \leq \int_{0}^{\infty} f_{2}(u) g(u) d u .
$$

Finally, we recall the definition of the Hardy-Littlewood-Pólya relation. Let $f$ and $g$ be locally integrable nonnegative functions on $\mathbb{R}_{+}$. We write $f \prec g$ if

$$
\int_{0}^{t} f(u) d u \leq \int_{0}^{t} g(u) d u
$$

for all $t>0$.

\section{THE LEVEL FUNCTION}

The notion of a level function was first introduced by Halperin [5. We shall use the extension of this notion given by Lorentz [12] and based on the following theorem.

Theorem 3.1. Let $\varphi$ be a positive measurable function on $\mathbb{R}_{+}$such that

$$
\Phi(t)=\int_{0}^{t} \varphi(u) d u<\infty
$$

for all $t>0$. Assume that $f$ is a nonnegative measurable function on $\mathbb{R}_{+}$and that

$$
\int_{0}^{t} f(u) d u=o(\Phi(t)) \text { as } t \rightarrow \infty .
$$

Then, there exists a nonnegative function $f^{\circ}$ on $\mathbb{R}_{+}$satisfying the following conditions:

(a) the function $f^{\circ}(t) / \varphi(t)$ decreases on $\mathbb{R}_{+}$;

(b) $f \prec f^{\circ}$;

(c) up to a set of measure zero, the set $\left\{t \in \mathbb{R}_{+}: f(t) \neq f^{\circ}(t)\right\}$ is the union of bounded disjoint intervals $I_{k}$ such that

$$
\int_{I_{k}} f(u) d u=\int_{I_{k}} f^{\circ}(u) d u
$$

and $f^{\circ}(t) / \varphi(t)$ is constant on $I_{k}$. 
This theorem is a slight modification of the results in [5] and [12, §3.6]; the proof is similar to the one given in [12, §3.6] for functions defined on $[0,1]$. It is easy to show that the function $f^{\circ}$ is uniquely determined (see [5, Theorem 3.7]). It is called the level function of $f$ with respect to $\varphi$.

Theorem 3.2. Let $1<p<\infty$ and $p<s \leq \infty$. Suppose that $f \in L^{p, s}\left(\mathbb{R}_{+}\right)$is a nonnegative and non-increasing function on $\mathbb{R}_{+}$. Let $f^{\circ}$ be the level function of $f$ with respect to the function $\varphi_{0}(t)=t^{-\alpha}, \alpha=1-s^{\prime} / p^{\prime}$. Then

$$
\left\|f^{\circ}\right\|_{p, s} \leq\|f\|_{p, s} \leq c_{p, s}\left\|f^{\circ}\right\|_{p, s}
$$

where

$$
c_{p, s}=\left(\frac{p}{s}\right)^{1 / s}\left(\frac{p^{\prime}}{s^{\prime}}\right)^{1 / s^{\prime}}
$$

The constants in the inequalities (3.1) are optimal.

Proof. First we assume that $s<\infty$. We consider the left hand side inequality in (3.1). Applying Theorem 3.1(c), we have $f^{\circ}(t)=\lambda_{k} t^{-\alpha}$ for all $t \in I_{k}$, where

$$
\lambda_{k}=\left(\int_{I_{k}} t^{-\alpha} d t\right)^{-1} \int_{I_{k}} f(t) d t
$$

Since $\alpha=(s / p-1) /(s-1)$ and $f^{\circ}(t)^{s-1} t^{s / p-1}=\lambda_{k}^{s-1}$, upon applying Hölder's inequality we obtain

$$
\begin{aligned}
\int_{I_{k}} f^{\circ}(t)^{s} t^{s / p-1} d t & =\lambda_{k}^{s-1} \int_{I_{k}} f^{\circ}(t) d t=\left(\int_{I_{k}} t^{-\alpha} d t\right)^{1-s}\left(\int_{I_{k}} f(t) d t\right)^{s} \\
& \leq \int_{I_{k}} f(t)^{s} t^{s / p-1} d t
\end{aligned}
$$

This estimate and property (c) yield the first inequality in (3.1).

Now, define

$$
\psi(t)=f(t)^{s-1} t^{s / p-1}
$$

Let $\widetilde{\psi}(t)$ be the level function of $\psi$ with respect to $\varphi(t)=1$. Applying Theorem 3.1 . Lemma 2.11, and the inequality (1.4), we obtain

$$
\begin{aligned}
\|f\|_{p, s}^{s} & =\int_{0}^{\infty} f(t) \psi(t) d t \leq \int_{0}^{\infty} f(t) \widetilde{\psi}(t) d t \\
& \leq \int_{0}^{\infty} f^{\circ}(t) \widetilde{\psi}(t) d t \leq\left\|f^{\circ}\right\|_{p, s}\|\widetilde{\psi}\|_{p^{\prime}, s^{\prime}}
\end{aligned}
$$

To obtain the second inequality in (3.1), it suffices to prove that

$$
\|\widetilde{\psi}\|_{p^{\prime}, s^{\prime}} \leq c_{p, s}\|f\|_{p, s}^{s-1}
$$

where the constant $c_{p, s}$ is defined by (3.2).

Let $E=\left\{t \in \mathbb{R}_{+}: \widetilde{\psi}(t)=\psi(t)\right\}$. Then, up to a set of measure zero,

$$
\mathbb{R}_{+} \backslash E=\bigcup_{k}\left(a_{k}, b_{k}\right)
$$

where $\left(a_{k}, b_{k}\right)$ are bounded disjoint intervals such that

$$
\widetilde{\psi}(t)=\frac{1}{b_{k}-a_{k}} \int_{a_{k}}^{b_{k}} \psi(u) d u, \quad \text { for all } t \in\left(a_{k}, b_{k}\right) .
$$


By Hölder's inequality,

$$
\begin{aligned}
\int_{a_{k}}^{b_{k}} \psi(u) d u & \leq\left(\int_{a_{k}}^{b_{k}} u^{s / p-1} d u\right)^{1 / s}\left(\int_{a_{k}}^{b_{k}} f(u)^{s} u^{s / p-1} d u\right)^{1 / s^{\prime}} \\
& =\left(\frac{p}{s}\right)^{1 / s}\left(b_{k}^{s / p}-a_{k}^{s / p}\right)^{1 / s}\left(\int_{a_{k}}^{b_{k}} f(u)^{s} u^{s / p-1} d u\right)^{1 / s^{\prime}} .
\end{aligned}
$$

Using (3.6) and applying Lemma 2.3, we obtain that

$$
\widetilde{\psi}(t) \leq\left(\frac{p}{s}\right)^{1 / s}\left(b_{k}^{s^{\prime} / p^{\prime}}-a_{k}^{s^{\prime} / p^{\prime}}\right)^{-1 / s^{\prime}}\left(\int_{a_{k}}^{b_{k}} f(u)^{s} u^{s / p-1} d u\right)^{1 / s^{\prime}},
$$

for all $t \in\left(a_{k}, b_{k}\right)$. Thus,

$$
\begin{aligned}
\int_{a_{k}}^{b_{k}} \widetilde{\psi}(t)^{s^{\prime}} t^{s^{\prime} / p^{\prime}-1} d t \leq & \left(\frac{p}{s}\right)^{s^{\prime} / s}\left(b_{k}^{s^{\prime} / p^{\prime}}-a_{k}^{s^{\prime} / p^{\prime}}\right)^{-1} \\
& \times \int_{a_{k}}^{b_{k}} f(t)^{s} t^{s / p-1} d t \int_{a_{k}}^{b_{k}} t^{s^{\prime} / p^{\prime}-1} d t \\
= & \left(\frac{p}{s}\right)^{s^{\prime} / s} \frac{p^{\prime}}{s^{\prime}} \int_{a_{k}}^{b_{k}} f(t)^{s} t^{s / p-1} d t .
\end{aligned}
$$

We also have that

$$
\begin{aligned}
\int_{E} \widetilde{\psi}(t)^{s^{\prime}} t^{s^{\prime} / p^{\prime}-1} d t & =\int_{E} \psi(t)^{s^{\prime}} t^{s^{\prime} / p^{\prime}-1} d t \\
& =\int_{E} f(t)^{s} t^{s / p-1} d t .
\end{aligned}
$$

Since

$$
c_{p, s}=\left(\frac{p}{s}\right)^{1 / s}\left(\frac{p^{\prime}}{s^{\prime}}\right)^{1 / s^{\prime}}>1,
$$

we obtain (3.5). Thus, the inequalities in (3.1) are proved for $s<\infty$.

Now let $s=\infty$ and hence $\alpha=1 / p$. We may assume that $f^{*}(t) t^{1 / p} \rightarrow 0$, as $t \rightarrow \infty$. Now let $I_{k}=\left(a_{k}, b_{k}\right)$. For any $k$,

$$
\begin{aligned}
p^{\prime}\left(b_{k}^{1 / p^{\prime}}-a_{k}^{1 / p^{\prime}}\right) \lambda_{k} & =\int_{a_{k}}^{b_{k}} f^{\circ}(t) d t=\int_{a_{k}}^{b_{k}} f(t) d t \\
& \leq\|f\|_{p, \infty} \int_{a_{k}}^{b_{k}} t^{-1 / p} d t=p^{\prime}\left(b_{k}^{1 / p^{\prime}}-a_{k}^{1 / p^{\prime}}\right)\|f\|_{p, \infty} .
\end{aligned}
$$

Thus, $\lambda_{k} \leq\|f\|_{p, \infty}$, which implies that $\left\|f^{\circ}\right\|_{p, \infty} \leq\|f\|_{p, \infty}$. On the other hand, for any $t \in\left(a_{k}, b_{k}\right)$ we have (see Theorem 3.1(b))

$$
t^{1 / p} f(t) \leq t^{1 / p-1} \int_{0}^{t} f(u) d u \leq t^{1 / p-1} \int_{0}^{t} f^{\circ}(u) d u \leq p^{\prime}\left\|f^{\circ}\right\|_{p, \infty} .
$$

This implies the second inequality in (3.1) for $s=\infty$.

The left hand side inequality in (3.1) becomes an equality for $f(t)=t^{-\alpha} \chi_{[0,1]}(t)$. Further, let $f=\chi_{[0,1]}$. Then

$$
\|f\|_{p, s}=\left(\frac{p}{s}\right)^{1 / s}
$$


Next, $f^{\circ}(t)=(1-\alpha) t^{-\alpha} \chi_{[0,1]}(t)$

$$
\left\|f^{\circ}\right\|_{p, s}=\left(\frac{s^{\prime}}{p^{\prime}}\right)^{1 / s^{\prime}}
$$

and we have the equality $\|f\|_{p, s}=c_{p, s}\left\|f^{\circ}\right\|_{p, s}$. Thus, the constants in (3.1) are optimal.

Remark 3.3. Let $1<p<s \leq \infty$. Let $f \in L^{p, s}\left(\mathbb{R}_{+}\right)$be a nonnegative and nonincreasing function on $\mathbb{R}_{+}$and let $f^{\circ}$ be the level function of $f$ with respect to the function $\varphi_{\alpha}(t)=t^{-\alpha}\left(\alpha=1-s^{\prime} / p^{\prime}\right)$. Then, the equality

$$
\left\|f^{\circ}\right\|_{p, s}=\|f\|_{p, s}
$$

holds if and only if $f^{\circ}(t)=f(t)$, except for a countable set of points $t$. Indeed, the last inequality in (3.3) becomes an equality if and only if $f(t) t^{\alpha}$ is constant on $I_{k}$.

In other words, (3.7) holds if and only if $f(t) t^{\alpha}$ decreases on $\mathbb{R}_{+}$.

\section{THE DUAL NORM}

Recall that for a function $f \in L^{p, s}(R, \mu)(1<p<\infty, 1 \leq s \leq \infty)$ its dual norm is defined by

$$
\|f\|_{p, s}^{\prime}=\sup \left\{\int_{R} f g d \mu: \quad\|g\|_{p^{\prime}, s^{\prime}}=1\right\},
$$

where the supremum is taken over all functions $g \in L^{p^{\prime}, s^{\prime}}(R, \mu)$ with $\|g\|_{p^{\prime}, s^{\prime}}=1$.

By Lemma 2.5 and the Hardy-Littlewood inequality [1, p. 44], for any function $f \in L^{p, s}(R, \mu)(1<p<\infty, 1 \leq s \leq \infty)$,

$$
\|f\|_{p, s}^{\prime}=\sup \left\{\int_{0}^{\infty} f^{*}(t) g(t) d t:\|g\|_{p^{\prime}, s^{\prime}}=1\right\}
$$

where the supremum is taken over all nonnegative and non-increasing functions $g \in L^{p^{\prime}, s^{\prime}}\left(\mathbb{R}_{+}\right)$with $\|g\|_{p^{\prime}, s^{\prime}}=1$.

Suppose that $1<p<\infty$ and $1 \leq s \leq \infty$. Let $f \in L^{p, s}\left(\mathbb{R}_{+}\right)$and let $g \in$ $L^{p^{\prime}, s^{\prime}}\left(\mathbb{R}_{+}\right)$. By Hölder's inequality (1.4),

$$
\int_{0}^{\infty}|f(t) g(t)| d t \leq\|f\|_{p, s}|| g \|_{p^{\prime}, s^{\prime}}
$$

It follows that

$$
\|f\|_{p, s}^{\prime} \leq\|f\|_{p, s}
$$

If $s \leq p$, then we have the equality of norms

$$
\|f\|_{p, s}^{\prime}=\|f\|_{p, s}
$$

Indeed,

$$
\|f\|_{p, s}^{s}=\int_{0}^{\infty} f^{*}(t) \psi(t) d t, \quad \psi(t)=f^{*}(t)^{s-1} t^{s / p-1} .
$$

If $s \leq p$, then the function $\psi$ is non-increasing and we have

$$
\|\psi\|_{p^{\prime}, s^{\prime}}^{s^{\prime}}=\int_{0}^{\infty} \psi(t)^{s^{\prime}} t^{s^{\prime} / p^{\prime}-1} d t=\|f\|_{p, s}^{s} .
$$

The latter two equalities imply that $\|f\|_{p, s}^{\prime} \geq\|f\|_{p, s}$. Together with (4.4) this yields (4.5). Observe also that the supremum in (4.2) is attained on the function $g(t)=\psi(t) /\|\psi\|_{p^{\prime}, s^{\prime}}$. 
Now we assume that $p<s \leq \infty$. Let $f \in L^{p, s}\left(\mathbb{R}_{+}\right)$. If the function $f^{*}(t) t^{1-s^{\prime} / p^{\prime}}$ is non-increasing, then as above we have the equality (4.5). Let $f$ be an arbitrary nonnegative function in $L^{p, s}\left(\mathbb{R}_{+}\right)$and let $g \in L^{p^{\prime}, s^{\prime}}\left(\mathbb{R}_{+}\right), g \geq 0$, be a non-increasing function. By Lemma 2.11, we have that

$$
\int_{0}^{\infty} f(t) g(t) d t \leq \inf _{f \prec h}\|h\|_{p, s}\|g\|_{p^{\prime}, s^{\prime}} .
$$

This implies that

$$
\|f\|_{p, s}^{\prime} \leq \inf _{f \prec h}\|h\|_{p, s} .
$$

Note that in the case where $s \leq p$ the infimum in (4.7) is equal to $\|f\|_{p, s}$. However, for $s>p$ the infimum may be smaller than $\|f\|_{p, s}$ and (4.6) may give a refinement of the inequality (4.3). It was proved by Halperin [5, Theorem 4.2] (see also [12, Theorem 3.6.5]) that equality in (4.7) holds and the infimum is attained for some $h \in L^{p, s}\left(\mathbb{R}_{+}\right)$. Since the proofs given in [5] and [12] do not cover explicitly the case $s=\infty$, and for the sake of completeness, we show the result for all $p<s \leq \infty$.

Theorem 4.1. Let $1<p<s \leq \infty$. Assume that $f \in L^{p, s}\left(\mathbb{R}_{+}\right)$is a nonnegative and non-increasing function on $\mathbb{R}_{+}$. Set $\alpha=1-s^{\prime} / p^{\prime}$ and $\varphi_{\alpha}(t)=t^{-\alpha}$. Then

$$
\|f\|_{p, s}^{\prime}=\inf _{f \prec h}\|h\|_{p, s}=\left\|f^{\circ}\right\|_{p, s},
$$

where $f^{\circ}$ is the level function of $f$ with respect to the function $\varphi_{\alpha}$.

Proof. In view of (4.7) and Theorem 3.1(b), it suffices to prove that

$$
\|f\|_{p, s}^{\prime} \geq\left\|f^{\circ}\right\|_{p, s} .
$$

Set

$$
E=\left\{x \in \mathbb{R}_{+}: f(x)=f^{\circ}(x)\right\} .
$$

By Theorem 3.1, up to a set of measure zero,

$$
\mathbb{R}^{+} \backslash E=\bigcup_{k}\left(a_{k}, b_{k}\right),
$$

where $\left(a_{k}, b_{k}\right)$ are disjoint bounded intervals such that

$$
\int_{a_{k}}^{b_{k}} f(t) d t=\int_{a_{k}}^{b_{k}} f^{\circ}(t) d t
$$

We first assume that $s<\infty$. Let $\psi(t)=f^{\circ}(t)^{s-1} t^{s / p-1}$. As above, we have

$$
\|\psi\|_{p^{\prime}, s^{\prime}}^{s^{\prime}}=\int_{0}^{\infty} \psi(t)^{s^{\prime}} t^{s^{\prime} / p^{\prime}-1} d t=\left\|f^{\circ}\right\|_{p, s}^{s}
$$

Set $g(t)=\psi(t) /\left\|f^{\circ}\right\|_{p, s}^{s-1}$. Then $\|g\|_{p^{\prime}, s^{\prime}}=1$. For each $k$, we have $f^{\circ}(t)=\lambda_{k} t^{-\alpha}$ and $\psi(t)=\lambda_{k}^{s-1}$, for $t \in\left(a_{k}, b_{k}\right)$ (where $\lambda_{k}$ is a constant). Thus,

$$
\begin{aligned}
\left\|f^{\circ}\right\|_{p, s}^{s-1} \int_{a_{k}}^{b_{k}} f(t) g(t) d t & =\lambda_{k}^{s-1} \int_{a_{k}}^{b_{k}} f(t) d t \\
& =\lambda_{k}^{s-1} \int_{a_{k}}^{b_{k}} f^{\circ}(t) d t=\int_{a_{k}}^{b_{k}}\left[t^{1 / p} f^{\circ}(t)\right]^{s} \frac{d t}{t} .
\end{aligned}
$$

Besides, we have

$$
\left\|f^{\circ}\right\|_{p, s}^{s-1} \int_{E} f(t) g(t) d t=\int_{E}\left[t^{1 / p} f^{\circ}(t)\right]^{s} \frac{d t}{t}
$$


and thus,

$$
\int_{0}^{\infty} f(t) g(t) d t=\left\|f^{\circ}\right\|_{p, s}
$$

from which we obtain (4.9).

Now let $s=\infty$. In this case we have

$$
\left\|f^{\circ}\right\|_{p, \infty}=\lim _{t \rightarrow 0+} f^{\circ}(t) t^{1 / p} .
$$

We assume first that for some $k$ we have $a_{k}=0$. Set

$$
g(t)=\chi_{\left(0, b_{k}\right)} /\left(p^{\prime} b_{k}^{1 / p^{\prime}}\right) .
$$

Then $\|g\|_{p^{\prime}, 1}=1$. We have

$$
f^{\circ}(t)=\lambda_{k} t^{-1 / p} \quad \text { for } \quad t \in\left(0, b_{k}\right) \text { and } \quad\left\|f^{\circ}\right\|_{p, \infty}=\lambda_{k} .
$$

Thus,

$$
\begin{aligned}
\int_{0}^{\infty} f(t) g(t) d t & =\left(p^{\prime} b_{k}^{1 / p^{\prime}}\right)^{-1} \int_{0}^{b_{k}} f(t) d t \\
& =\left(p^{\prime} b_{k}^{1 / p^{\prime}}\right)^{-1} \int_{0}^{b_{k}} f^{\circ}(t) d t=\lambda_{k}=\left\|f^{\circ}\right\|_{p, \infty} .
\end{aligned}
$$

This implies (4.9).

Now we assume that $a_{k} \neq 0$ for each $k$. Then, for any $\delta>0$ we have

$$
(0, \delta) \cap A \neq \emptyset, \quad \text { where } \quad A=\mathbb{R}_{+} \backslash \bigcup_{j}\left(a_{j}, b_{j}\right) .
$$

On the other hand, by Theorem 3.1 (c), for any $t \in A$,

$$
\int_{0}^{t} f(u) d u=\int_{0}^{t} f^{\circ}(u) d u .
$$

Let $\varepsilon>0$. By (4.11), there exists $\delta>0$ such that

$$
f^{\circ}(t) t^{1 / p}>\left\|f^{\circ}\right\|_{p, \infty}-\varepsilon \quad \text { for any } t \in(0, \delta) .
$$

Let $\xi \in(0, \delta) \cap A$. Set $g(t)=\chi_{(0, \xi)} /\left(p^{\prime} \xi^{1 / p^{\prime}}\right)$. Then $\|g\|_{p^{\prime}, 1}=1$. Applying (4.13) and (4.12), we get

$$
\int_{0}^{\infty} f(t) g(t) d t=\left(p^{\prime} \xi^{1 / p^{\prime}}\right)^{-1} \int_{0}^{\xi} f^{\circ}(t) d t>\left\|f^{\circ}\right\|_{p, \infty}-\varepsilon,
$$

which again implies (4.9).

Remark 4.2. Note that for $1<p<s<\infty$ the supremum in (4.2) is attained on the function $g(t)=\psi(t) /\|\psi\|_{p^{\prime}, s^{\prime}}$, where $\psi(t)=f^{\circ}(t)^{s-1} t^{s / p-1}$. If $s=\infty$, then the supremum in (4.2) may not be attained.

Remark 4.3. Let $1<p<s \leq \infty$, and let $f \in L^{p, s}\left(\mathbb{R}_{+}\right)$be a nonnegative and non-increasing function on $\mathbb{R}_{+}$. Then, by Remark 3.3, the equality

$$
\|f\|_{p, s}^{\prime}=\|f\|_{p, s}
$$

holds if and only if $f(t) t^{\alpha}$ decreases on $\mathbb{R}_{+}$. 
The following theorem gives the sharp estimate of the standard norm via the dual norm.

Theorem 4.4. Let $1<p<\infty$ and $p<s \leq \infty$. Then, for any function $f \in$ $L^{p, s}(R, \mu)$,

$$
\|f\|_{p, s} \leq c_{p, s}\|f\|_{p, s}^{\prime}
$$

where

$$
c_{p, s}=\left(\frac{p}{s}\right)^{1 / s}\left(\frac{p^{\prime}}{s^{\prime}}\right)^{1 / s^{\prime}}
$$

The constant $c_{p, s}$ is optimal.

This theorem follows immediately from Theorems 4.1 and 3.2. However, a direct proof can be given exactly as in Theorem 3.2. Indeed, assume that $f$ is nonnegative and non-increasing on $\mathbb{R}_{+}$. As in the proof of Theorem 3.2 we have

$$
\begin{aligned}
\|f\|_{p, s}^{s} & =\int_{0}^{\infty} f(t) \psi(t) d t \leq \int_{0}^{\infty} f(t) \widetilde{\psi}(t) d t \\
& \leq\|f\|_{p, s}^{\prime}\|\widetilde{\psi}\|_{p^{\prime}, s^{\prime}} .
\end{aligned}
$$

Applying the inequality (3.5), we obtain (4.14). Let $f=\chi_{[0,1]}$. Then, by Lemma 2.10.

$$
\|f\|_{p, s}^{\prime}=\left(\frac{s^{\prime}}{p^{\prime}}\right)^{1 / s^{\prime}} \text { and }\|f\|_{p, s}=\left(\frac{p}{s}\right)^{1 / s}=c_{p, s}\|f\|_{p, s}^{\prime},
$$

which shows that the constant in (4.14) is optimal.

\section{THE DECOMPOSITION NORM}

In this section we prove one of the main results of this paper-the coincidence of the dual and the decomposition norms. The following lemma plays an important role in the proof of the equality of these two norms.

Lemma 5.1. Let $\alpha_{1} \geq \alpha_{2} \geq \cdots \geq \alpha_{\nu}$ be positive numbers and let $\left\{\eta_{j k}\right\}$ be a $(N \times \nu)$-matrix of positive numbers $(1 \leq j \leq N, 1 \leq k \leq \nu)$. Set

$$
\beta_{k}=\sum_{j=1}^{N} \eta_{j k}, \quad k=1, \cdots, \nu .
$$

Assume that

$$
\beta_{1}+\cdots+\beta_{k} \geq \alpha_{1}+\cdots+\alpha_{k}
$$

for any $k=1, \ldots, \nu$. Let $\eta=\max \eta_{j k}$. Then, for any $j=1, \ldots, N$ there exists a permutation $\left\{\tilde{\eta}_{j k}\right\}_{k=1}^{\nu}$ of the $\nu$-tuple $\left\{\eta_{j k}\right\}_{k=1}^{\nu}$ such that

$$
\alpha_{k} \leq \tilde{\beta}_{k}+\eta, \quad \tilde{\beta}_{k}=\sum_{j=1}^{N} \tilde{\eta}_{j k},
$$

for any $k=1, \ldots, \nu$. 
Proof. For $\nu=1$ the lemma is obvious. Assume that it is true for $\nu-1(\nu \geq 2)$. We have $\beta_{1} \geq \alpha_{1}$. If $\beta_{k} \geq \alpha_{1}$ for all $k=1, \ldots, \nu$, there is nothing to prove. Otherwise, denote by $s$ the smallest natural number $k$ for which $\beta_{k}<\alpha_{1}$. Then, $s \geq 2$. Set $\gamma_{0}=\beta_{1}, \gamma_{N}=\beta_{s}$, and

$$
\gamma_{m}=\sum_{j=1}^{m} \eta_{j s}+\sum_{j=m+1}^{N} \eta_{j 1}, \quad \text { for } \quad 1 \leq m<N .
$$

We have $\gamma_{0} \geq \alpha_{1}$ and $\gamma_{N}<\alpha_{1}$. Let $m_{0}$ be the smallest $m$ for which $\gamma_{m}<\alpha_{1}$. Since $\left|\gamma_{m}-\gamma_{m-1}\right| \leq \eta$ for any $m=1, \cdots, N$, we have that

$$
\gamma_{m_{0}}<\alpha_{1} \leq \gamma_{m_{0}-1} \leq \gamma_{m_{0}}+\eta
$$

Set

$$
\begin{aligned}
& \tilde{\eta}_{j 1}= \begin{cases}\eta_{j s} & \text { if } 1 \leq j \leq m_{0} \\
\eta_{j 1} & \text { if } m_{0}<j \leq N,\end{cases} \\
& \eta_{j s}^{\prime}= \begin{cases}\eta_{j 1} & \text { if } 1 \leq j \leq m_{0} \\
\eta_{j s} & \text { if } m_{0}+1<j \leq N,\end{cases}
\end{aligned}
$$

and $\eta_{j k}^{\prime}=\eta_{j k}(j=1, \ldots, N)$ if $k \neq 1, s$. Using (5.3) we have

$$
\tilde{\beta}_{1}<\alpha_{1} \leq \tilde{\beta}_{1}+\eta, \quad \text { where } \quad \tilde{\beta}_{1}=\gamma_{m_{0}}=\sum_{j=1}^{N} \eta_{j s}^{\prime} .
$$

Also let $\beta_{k}^{\prime}=\beta_{k}$ for $k=2, \ldots, N$. We first assume that $s=2$. We have

$$
\beta_{1}+\beta_{2}+\cdots+\beta_{k} \geq \alpha_{1}+\alpha_{2}+\cdots+\alpha_{k},
$$

for each $k \geq 2$. But $\beta_{1}+\beta_{2}=\tilde{\beta}_{1}+\beta_{2}^{\prime}$ and $\tilde{\beta}_{1}<\alpha_{1}$, by (5.4). Thus,

$$
\beta_{2}^{\prime}+\cdots+\beta_{k}^{\prime} \geq \alpha_{2}+\cdots+\alpha_{k}, \quad k=2, \ldots, \nu .
$$

Now we assume that $s>2$. Then we have, for every $2 \leq l<s$,

$$
\beta_{l}^{\prime}=\beta_{l} \geq \alpha_{1} \geq \alpha_{l}
$$

and therefore

$$
\beta_{2}^{\prime}+\cdots+\beta_{k}^{\prime} \geq \alpha_{2}+\cdots+\alpha_{k}, \quad 2 \leq k<s .
$$

Let $k \geq s$. Since

$$
\tilde{\beta}_{1}+\beta_{2}^{\prime}+\cdots+\beta_{k}^{\prime}=\beta_{1}+\beta_{2}+\cdots+\beta_{k}
$$

and $\tilde{\beta}_{1}<\alpha_{1}$ (see (5.4)), it follows from (5.1) that

$$
\beta_{2}^{\prime}+\cdots+\beta_{k}^{\prime} \geq \alpha_{2}+\cdots+\alpha_{k} .
$$

Thus, we can apply our inductive assumption to the $(N \times(\nu-1))$-matrix

$$
\left\{\eta_{j k}^{\prime}\right\}, \quad 1 \leq j \leq N, 2 \leq k \leq \nu .
$$

Together with (5.4), this proves the lemma.

Theorem 5.2. Let $1<p<\infty$ and $1 \leq s \leq \infty$. Then, for any function $f \in$ $L^{p, s}(R, \mu)$,

$$
\|f\|_{p, s}^{\prime}=\|f\|_{(p, s)} .
$$


Proof. If $s \leq p$, then

$$
\|f\|_{p, s}^{\prime}=\|f\|_{p, s}=\|f\|_{(p, s)} .
$$

We assume that $1<p<s \leq \infty$. By Lemma 2.6.

$$
\|f\|_{p, s}^{\prime} \leq\|f\|_{(p, s)} \text {. }
$$

We shall prove that

$$
\|f\|_{(p, s)} \leq\|f\|_{p, s}^{\prime} .
$$

By virtue of (2.5) and (2.9), it suffices to prove (5.6) in the case where $(R, \mu)$ is $\mathbb{R}_{+}$with Lebesgue's measure and $f$ is a nonnegative and non-increasing function on $\mathbb{R}_{+}$. Applying Lemma 2.7(3), we can also assume that there exist $0<x_{0}<x_{1}<\infty$ such that $f(x)=c_{0}>0$ on $\left(0, x_{0}\right)$ and $f(x)=0$ for all $x>x_{1}$.

By Theorem 4.1 ,

$$
\|f\|_{p, s}^{\prime}=\left\|f^{\circ}\right\|_{p, s}
$$

where $f^{\circ}$ is the level function of $f$ with respect to the function $\varphi_{\alpha}(t)=t^{-\alpha}, \alpha=$ $1-s^{\prime} / p^{\prime}$. Set

$$
E=\left\{x \in \mathbb{R}_{+}: f(x)=f^{\circ}(x)\right\} .
$$

By Theorem 3.1 up to a set of measure zero,

$$
\mathbb{R}_{+} \backslash E=\bigcup_{i}\left(a_{i}, b_{i}\right),
$$

where $\left(a_{i}, b_{i}\right)$ are disjoint bounded intervals such that

$$
\int_{a_{i}}^{x} f(t) d t \leq \int_{a_{i}}^{x} f^{\circ}(t) d t, \quad x \in\left(a_{i}, b_{i}\right)
$$

and

$$
\int_{a_{i}}^{b_{i}} f(t) d t=\int_{a_{i}}^{b_{i}} f^{\circ}(t) d t .
$$

By our assumption, $f(x)=c_{0}$ on $\left(0, x_{0}\right)$. At the same time, $f^{\circ}$ is strictly decreasing on $\left(0, x_{0}\right)$. This implies that for some $i$ we have $a_{i}=0$. Indeed, assume the contrary. Then, as is easily seen, there exists $\left(a_{k}, b_{k}\right)$ such that $0<a_{k}<b_{k}<x_{0}$. We have $f^{\circ}(x)=\lambda_{k} x^{-\alpha}$ on $\left(a_{k}, b_{k}\right)$. Further,

$$
c_{0} a_{k}=\int_{0}^{a_{k}} f^{\circ}(x) d x>\frac{\lambda_{k} a_{k}^{1-\alpha}}{1-\alpha},
$$

and therefore

$$
\lambda_{k}<c_{0}(1-\alpha) a_{k}^{\alpha} .
$$

From here,

$$
\begin{aligned}
c_{0} b_{k} & =\int_{0}^{b_{k}} f^{\circ}(x) d x=c_{0} a_{k}+\int_{a_{k}}^{b_{k}} f^{\circ}(x) d x \\
& =c_{0} a_{k}+\frac{\lambda_{k}}{1-\alpha}\left(b_{k}^{1-\alpha}-a_{k}^{1-\alpha}\right)<c_{0} b_{k} .
\end{aligned}
$$

Thus, we can assume that $a_{1}=0$. Let $b=\max \left(x_{1}, \sup _{j} b_{j}\right)$. Then $b<\infty$ and $f^{\circ}(x)=0$ for all $x>b$.

Let $\varepsilon>0$. For any $\nu \in \mathbb{N}$, define the function $g_{\nu}$ in the following way. First, set $g_{\nu}(x)=f(x)$ for $x \in E$; then $g_{\nu}(x)=0$ for all $x>b$. Further, we subdivide each 
interval $\left(a_{i}, b_{i}\right)$ into $\nu$ subintervals $\Delta_{k}^{i}, k=1, \cdots, \nu$, of length $\left|\Delta_{k}^{i}\right|=\left(b_{i}-a_{i}\right) / \nu$, and set

$$
g_{\nu}(x)=\left|\Delta_{k}^{i}\right|^{-1} \int_{\Delta_{k}^{i}} f(t) d t \quad \text { for } \quad x \in \Delta_{k}^{i}, \quad k=1, \ldots, \nu .
$$

It is easy to see that there exists $\nu_{1}$ such that

$$
\left\|f-g_{\nu}\right\|_{p, s}<\varepsilon
$$

for all $\nu \geq \nu_{1}$. It follows that, for all $\nu \geq \nu_{1}$,

$$
\begin{aligned}
\|f\|_{(p, s)} & \leq\left\|g_{\nu}\right\|_{(p, s)}+\left\|f-g_{\nu}\right\|_{p, s} \\
& \leq\left\|g_{\nu}\right\|_{(p, s)}+\varepsilon .
\end{aligned}
$$

Similarly, for every $\nu \in \mathbb{N}$ we define the function $\psi_{\nu}$ approximating $f^{\circ}$. Set $\psi_{\nu}(x)=f^{\circ}(x)$ for $x \in E$ and

$$
\psi_{\nu}(x)=\left|\Delta_{k}^{i}\right|^{-1} \int_{\Delta_{k}^{i}} f^{\circ}(t) d t \quad \text { for } \quad x \in \Delta_{k}^{i}, \quad k=1, \ldots, \nu .
$$

There exists an integer $\nu_{2} \geq \nu_{1}$ such that

$$
\left\|\psi_{\nu}\right\|_{p, s} \leq\left\|f^{\circ}\right\|_{p, s}+\varepsilon
$$

for all $\nu \geq \nu_{2}$. Fix $\nu \geq \nu_{2}$. Next, choose a number $\delta>0$ such that

$$
\delta<\varepsilon b^{-1 / p}\left(\frac{s}{p}\right)^{1 / s} .
$$

We shall prove that there exist a number $N \in \mathbb{N}$ and functions $f_{j} \geq 0, j=1, \ldots, N$, such that

$$
g_{\nu}(x) \leq \sum_{j=1}^{N} f_{j}(x)+\delta, \quad x>0
$$

and

$$
\left\|f_{j}\right\|_{p, s}=\left\|\psi_{\nu}\right\|_{p, s} / N, \quad j=1, \ldots, N
$$

For any $i$, define

$$
\beta_{k}^{(i)}=\left|\Delta_{k}^{i}\right|^{-1} \int_{\Delta_{k}^{i}} f^{\circ}(t) d t, \quad k=1, \ldots, \nu .
$$

There exists a number $N^{\prime} \in \mathbb{N}$ such that

$$
\beta_{k}^{(1)}<N^{\prime} \delta, \quad k=1, \ldots, \nu .
$$

On the other hand, since $f^{\circ}$ is bounded on $\left[b_{1}, \infty\right)$, there exists $N^{\prime \prime} \in \mathbb{N}$ such that for all $i \geq 2$,

$$
\beta_{k}^{(i)}<N^{\prime \prime} \delta, \quad k=1, \ldots, \nu .
$$

Let $N=\max \left(N^{\prime}, N^{\prime \prime}\right)$. Then, for any $i$,

$$
\beta_{k}^{(i)}<N \delta, \quad k=1, \ldots, \nu
$$

Now we define the functions $f_{j}, j=1, \ldots, N$. Set

$$
f_{j}(x)=\frac{1}{N} \psi_{\nu}(x), \quad \text { for } \quad x \in E .
$$


Further, consider an interval $\left(a_{i}, b_{i}\right)$. Set

Let

$$
\eta_{j k}^{(i)}=\frac{\beta_{k}^{(i)}}{N}, \quad j=1, \ldots, N, \quad k=1, \ldots, \nu
$$

Then, by (5.7),

$$
\alpha_{k}^{(i)}=\left|\Delta_{k}^{i}\right|^{-1} \int_{\Delta_{k}^{i}} f(t) d t
$$

$$
\beta_{1}^{(i)}+\cdots+\beta_{k}^{(i)} \geq \alpha_{1}^{(i)}+\cdots+\alpha_{k}^{(i)}, \quad k=1, \ldots, \nu
$$

Applying Lemma 5.1, we obtain that for any fixed $i$ and every $j=1, \ldots, N$, there exists a permutation $\left\{\tilde{\eta}_{j k}^{(i)}\right\}_{k=1}^{\nu}$ of the $\nu$-tuple $\left\{\eta_{j k}^{(i)}\right\}_{k=1}^{\nu}$ such that

$$
\alpha_{k}^{(i)} \leq \sum_{j=1}^{N} \tilde{\eta}_{j k}^{(i)}+\delta
$$

for any $k=1, \ldots, \nu$. Set

$$
f_{j}(x)=\tilde{\eta}_{j k}^{(i)}, \quad \text { for } \quad x \in \Delta_{k}^{i} .
$$

The functions $f_{j}(j=1, \ldots, N)$ are defined on $\mathbb{R}_{+}$and each of them is equimeasurable with $\psi_{\nu} / N$. Thus, we have (5.14). Moreover, (5.15) implies (5.13). Applying (5.12) - (5.14) and taking into account the fact that $g_{\nu}(x)=0$ for $x>b$, we obtain

$$
\left\|g_{\nu}\right\|_{(p, s)} \leq \sum_{j=1}^{N}\left\|f_{j}\right\|_{p, s}+\delta\left\|\chi_{[0, b]}\right\|_{p, s} \leq\left\|\psi_{\nu}\right\|_{p, s}+2 \varepsilon .
$$

Using (5.10) and (5.11), we obtain

$$
\|f\|_{(p, s)} \leq\left\|f^{\circ}\right\|_{p, s}+4 \varepsilon .
$$

This implies (5.6).

Corollary 5.3. Let $f \in L^{p, s}(R, \mu)(1 \leq p<\infty, 1 \leq s \leq \infty)$. Then

$$
\|f\|_{(p, s)}=\left\|f^{*}\right\|_{(p, s)} .
$$

Indeed, (5.16) follows immediately from (2.5) and (5.5). Observe that (5.16) does not follow directly from the definition.

\section{The TRIANGLE INEQUALITY}

Applying Theorem 4.4 and Theorem [5.2, we immediately obtain the following version of the "triangle inequality".

Theorem 6.1. Let $1<p<s \leq \infty$. Assume that $f_{k} \in L^{p, s}(R, \mu)(k=1, \ldots, N)$. Then

$$
\left\|\sum_{k=1}^{N} f_{k}\right\|_{p, s} \leq c_{p, s} \sum_{k=1}^{N}\left\|f_{k}\right\|_{p, s}
$$

where

$$
c_{p, s}=\left(\frac{p}{s}\right)^{1 / s}\left(\frac{p^{\prime}}{s^{\prime}}\right)^{1 / s^{\prime}}
$$

and the constant is optimal. 
Remark 6.2. It is clear that (6.1) is equivalent to the inequality

$$
\|f\|_{p, s} \leq c_{p, s}\|f\|_{(p, s)}
$$

where $f$ is any function in $L^{p, s}(R, \mu)$. Inequality (6.2) follows directly from (4.14) and Lemma 2.6. By Lemma 2.10, (6.2) becomes equality for $f=\chi_{[0,1]}$. Thus, Theorem 6.1 follows from Theorem 4.4 and Lemmas 2.6 and 2.10

We also have the following continuous version of the Minkowski type inequality.

Theorem 6.3. Suppose that $(R, \mu)$ is a $\sigma$-finite nonatomic measure space and $(Q, \nu)$ is a $\sigma$-finite measure space. Let $f$ be a nonnegative measurable function on $(R \times Q, \mu \times \nu)$. Assume that $1<p<s \leq \infty$ and that for almost all $y \in Q$ the function

$$
f_{y}(x)=f(x, y), \quad x \in R,
$$

belongs to $L^{p, s}(R, \mu)$. Set $F(x)=\int_{Q} f(x, y) d \nu(y), x \in R$. Then

$$
\|F\|_{p, s} \leq c_{p, s} \int_{Q}\left\|f_{y}\right\|_{p, s} d \nu(y)
$$

where

$$
c_{p, s}=\left(\frac{p}{s}\right)^{1 / s}\left(\frac{p^{\prime}}{s^{\prime}}\right)^{1 / s^{\prime}}
$$

and the constant is optimal.

Proof. By Theorem 4.4 .

$$
\|f\|_{p, s} \leq c_{p, s}\|f\|_{p, s}^{\prime},
$$

where the constant $c_{p, s}$ is defined by (6.4). Let $g \in L^{p^{\prime}, s^{\prime}}(R, \mu)$ and assume that $\|g\|_{p^{\prime}, s^{\prime}}=1$. Applying Fubini's Theorem and Hölder's inequality, we obtain

$$
\begin{aligned}
\int_{R} F(x) g(x) d \mu(x) & =\int_{R}\left(\int_{Q} f(x, y) d \nu(y)\right) g(x) d \mu(x) \\
& =\int_{Q} \int_{R} f(x, y) g(x) d \mu(x) d \nu(y) \leq \int_{Q}\left\|f_{y}\right\|_{p, s} d \nu(y) .
\end{aligned}
$$

Together with (6.5), this implies (6.3). Finally, it follows from Theorem 6.1 that the constant $c_{p, s}$ in (6.3) cannot be replaced by a smaller one.

\section{REFERENCES}

[1] C. Bennett and R. Sharpley, Interpolation of Operators, Academic Press, Boston, 1988. MR928802 (89e:46001)

[2] M. J. Carro, J. A. Raposo, and J. Soria, Recent Developments in the Theory of Lorentz Spaces and Weighted Inequalities, Mem. Amer. Math. Soc. 187, Providence, RI, 2007. MR 2308059 (2008b:42034)

[3] M. J. Carro and J. Soria, Weighted Lorentz spaces and the Hardy operator, J. Funct. Anal. 112 (1993), 480-494. MR1213148 (94f:42025)

[4] E. DiBenedetto, Real Analysis, Birkhäuser, Boston, 2002. MR.1897317 (2003d:00001)

[5] I. Halperin, Function spaces, Canad. J. Math. 5 (1953), 273-288. MR0056195 (15:38h)

[6] H. P. Heinig and L. Maligranda, Chebyshev inequality in function spaces, Real Anal. Exchange 17 (1991-92), 211-247. MR.1147365 (92k:26045)

[7] R. A. Hunt, On L $(p, q)$, Enseignement Math. 12 (1966), 249-276. MR0223874 (36:6921)

[8] V. I. Kolyada, Rearrangement of functions and embedding of anisotropic spaces of Sobolev type, East J. Approx. 4 (1998), no. 2, 111-199. MR1638343 (99g:46043a) 
[9] V. I. Kolyada, Inequalities of Gagliardo-Nirenberg type and estimates for the moduli of continuity, Russian Math. Surveys 60 (2005), 1147-1164. MR2215758 (2007b:26026)

[10] G. G. Lorentz, Some new functional spaces, Ann. of Math. 51 (1950), 37-55. MR0033449 $(11: 442 \mathrm{~d})$

[11] G. G. Lorentz, On the theory of spaces $\Lambda$, Pacific J. Math. 1 (1951), 411-429. MR0044740 $(13: 470 \mathrm{c})$

[12] G. G. Lorentz, Bernstein polynomials, Univ. of Toronto Press, Toronto, 1953. MR0057370 $(15: 217 \mathrm{a})$

[13] E. Sawyer, Boundedness of classical operators on classical Lorentz spaces, Studia Math. 96 (1990), 145-158. MR1052631 (91d:26026)

[14] E. M. Stein and G. Weiss, Introduction to Fourier Analysis on Euclidean Spaces, Princeton Univ. Press, 1971. MR0304972 (46:4102)

Department of Mathematics, Karlstad University, SE-65188 Karlstad, Sweden

E-mail address: sorina.barza@kau.se

Department of Mathematics, Karlstad University, SE-65188 Karlstad, Sweden

E-mail address: viktor.kolyada@kau.se

Department of Applied Mathematics and Analysis, University of Barcelona, E-08007 Barcelona, Spain

E-mail address: soria@ub.edu 